\title{
Healthy brain ageing: the new concept of motivational reserve ${ }^{\dagger}$
}

\author{
Andreas Maercker, ${ }^{1}$ Simon Forstmeier ${ }^{2}$
}

The Psychiatrist (2011), 35, 175-177, doi: 10.1192/pb.bp.111.036830

${ }^{1}$ Department of Psychopathology, University of Zurich; ${ }^{2}$ Department of Psychology, University of Zurich

Correspondence to Andreas Maercker (maercker@psychologie.uzh.ch)

First received 7 Sep 2011, accepted 8 Sep 2011
Summary Various research approaches try to solve the puzzles related to dementia or healthy brain ageing. Neurogenetic, neuroimaging and neuropharmacological research are the most visible of these. Psychological research in dementia has been instrumental in improving familial or institutional care. However, it has not been regarded as basic research. Recent psychological research into the mechanisms of co-forming or shaping the clinical presentation of dementias can be counted as truly basic research. The 'cognitive or brain reserve' concept has been fruitful in implementing psychological knowledge on plasticity factors in dementia. The new concept of 'motivational reserve capacity' extends these psychological concepts. In particular, it provides a basis for integrating various findings on how lifestyle factors effectively shape clinical dementia presentations - or lead to healthy brain ageing.

\section{Declaration of interest None.}

As is well known, longer life expectancy leads to an increase in the prevalence of dementia. This means that our efforts to alleviate or at least defer the onset of Alzheimer's disease and other dementias must be all the more urgent.

The terms 'brain health', 'brain fitness' and 'improvement of lifestyle' are often used to refer to self-determined areas of cognitive ageing. Geropsychology and geropsychiatry constantly supply us with study results which seem to support the view that dementia is associated with everyday activities and lifestyles that affect our health.

In this paper, we explain the concept of 'motivational reserve' that complements the more established concepts of brain reserve and cognitive reserve. Brain reserve is believed to enable the brain to tolerate neuropathological dementiarelated changes that do not have clinical manifestations. ${ }^{1}$ The brain uses untended networks more efficiently and thus compensates for networks that are disrupted, which is an example of neuronal plasticity. Motivational reserve refers to the driving forces or processes that lead to this more efficient or flexible use of brain networks. We hypothesised that people with strong motivational abilities are at a lower risk of developing age-related cognitive decline or dementia. ${ }^{2,3}$ Motivational reserve thus equips the individual with resilience against neuropathological damage.

\section{Motivational reserve studies Delay of gratification studies}

Motivational abilities that contribute to motivational reserve are - in everyday language - self-discipline, willpower

†See commentary, pp. 178-179. and diligence. Motivation psychology extensively investigates concepts such as motivation regulation, decision regulation, activation regulation, goal orientation, intentionality and self-efficacy. Impressive accounts of the results of research on the delay of gratification have been presented at almost all developmental psychology meetings all over the world. One example would be studies with children conducted by the eminent psychologist Walter Mischel using the 'marshmallow test'. ${ }^{4}$ The children were asked to sit at a table on which a marshmallow was placed. They were told that they would be given two marshmallows if they waited for the researcher to arrive. About $75 \%$ of children were unable to wait, but $25 \%$ managed to, and were rewarded with two marshmallows. Astonishingly, this ability to delay gratification is a relatively stable personality attribute predictive of many later abilities of children and adolescents, such as intelligence, academic and social competence, selfcontrol, self-efficacy, goal orientation, as well as health-related behaviours such as drug misuse. ${ }^{5}$

\section{Role of motivational abilities in guarding against dementia}

Which motivational abilities therefore might be responsible for an increased resilience to dementia? No delay of gratification tests for adults or the elderly so far exist, although we are working on adequate complex variants of such a test. ${ }^{6}$ Adults differ with regard to motivational engagement in their work and other activities. Occupational scientists have developed a directory that provides a specific profile of motivational, cognitive and social abilities, among others, for a huge range of occupations (Occupational Information Network, $\left.\mathrm{O}^{*} \mathrm{NET}\right){ }^{7}$ For example, fashion 
designers show high motivational abilities, but retail salespersons show rather low motivational abilities.

The core assumption of the motivational reserve model is that a high motivational ability leads to a lower incidence of cognitive decline and dementia through a higher brain reserve, and we were able to apply our model to an existing longitudinal study. ${ }^{8}$ The German AgeCoDe sample ${ }^{9}$ comprises 3300 individuals aged 75 and above who do not have dementia; so far, they have been followed up twice, after 1.5 and 3 years. About 300 participants have developed mild cognitive impairment and 70 have developed Alzheimer's disease. It was found that high occupationrelated motivational abilities in mid-life reduced the risk of mild cognitive impairment by $35 \%$. The effect on the risk of Alzheimer's depended on the presence of an ApoE e4 allele which intensifies the biochemical disturbances characteristic of the disease, including beta-amyloid deposition, tangle formation, neuronal cell death and synaptic plasticity. Mid-life motivational abilities were associated with a reduced risk of Alzheimer's disease in ApoE e4 carriers but not in non-carriers. Confirming previous results from a cross-sectional study, ${ }^{2}$ this was now clear evidence supporting the assumption that high motivational abilities in mid-life have effects that both promote health and prevent cognitive decline in later life. These effects seem to go beyond pure cognitive abilities, such as verbal intelligence, education, and occupationrelated cognitive abilities in mid-life. ${ }^{8}$

\section{Motivational reserve and healthy brain}

What, therefore, are the consequences of healthy brain ageing? Similar results from other studies point in the same direction. Many original studies and meta-analyses show that the extent of physical activities (e.g. walking, dancing, swimming) and social activities (e.g. visiting friends, going to the theatre, participating in social groups) an individual engages in influences the incidence of dementia. ${ }^{3,10}$ Cognitive activities (e.g. reading books and newspapers, writing, studying, doing crossword puzzles) have long been credited with protective effects and have shown promising results in studies. In our opinion, a mixture of motivational ('I motivate myself to be active') and cognitive ('I remain mentally fit') factors always underlie these cognitive abilities. However, cognitive abilities have been traditionally considered more important than motivational (and social) factors, which are referred to in the established concept of cognitive reserve as the main resiliency factor against age-related decline. Two independent large-scale studies indicate the importance of motivational abilities.

The first study investigated 'conscientiousness', the personality trait that refers to an individual's tendency to control impulses and be goal directed. A cohort of 1000 men and women over 65 years old in religious orders in the USA were followed up 12 years after enrolment. ${ }^{11} \mathrm{~A}$ high level of conscientiousness was associated with an $89 \%$ reduction in the risk of Alzheimer's disease. The results were not substantially different when other personality traits, depression, activity patterns, vascular conditions, and other risk factors were taken into account. Interestingly, the level of conscientiousness is a subtle marker of clinically manifest Alzheimer's disease because people with clinically diagnosed Alzheimer's disease are described by others as being less conscientious than they were before onset, and further decline is observed as the disease progresses. ${ }^{12}$

\section{A purposeful life equals a healthier life}

Having a purpose in life also constitutes a motivational ability because it results in intentionality and goal-directed action that guides behaviour. Researchers in Chicago ${ }^{13}$ found that a high purpose in life, reported by people over 80 years old, reduced the incidence of mild cognitive impairment in the subsequent 7 years by a factor of 1.4 and the incidence of Alzheimer's disease by a factor of 2.4 (even when controlling for covariates such as education and depression). The authors found no causal explanation for this surprising finding, other than the fact that a purpose in life is generally associated with lower mortality and changes in a range of physical health parameters (e.g. increased high-density lipoprotein cholesterol levels, fewer pathological immune markers). Since purpose in life may translate into an increased sense of intentionality or goal-directed striving, in our view the result of the study contributes to the broader concept of motivational reserve.

Both conscientiousness and purpose in life do not arise late in life. Basic research has shown that these two factors and motivational abilities in general - gradually develop throughout young adulthood and old age, perhaps partly in response to life experiences and changing circumstances in the workplace and home environment that occur at those periods. Interestingly, in the Chicago study on 'purpose of life', ${ }^{13}$ no reference was made to the earlier study on conscientiousness, ${ }^{11}$ although we consider both variables closely related as motivational factors.

\section{Motivational resilience - clinical implications}

What can we infer from all this for healthy brain ageing and the prevention of age-related cognitive decline or an increase in the incidence of Alzheimer's disease? Is it possible that there are motivation-related interventions that may influence the functioning of the brain? When answering these questions, we must differentiate between two potential windows of opportunity for prevention or intervention: long-term prevention in mid-life and shortterm interventions in patients older than 70 . We are convinced that the focus of prevention should be in the mid-life period because these are rather new and important considerations.

Popular enthusiasm for some types of lifestyle interventions has been considerable. In particular, cognitive stimulation enjoys wide media coverage, ${ }^{14}$ although the benefits are not strictly evidence based. The ability to learn new things, which is certainly maintained in older age, is still viewed by some as the core factor in resilience against cognitive decline or neuropathology. However, it is conceivable that neurogenesis of dendrites or intensive brain activation might also be stimulated by a sense of purpose or drive, and striving. 
We feel it is time to reconsider the role of psychologically inspired lifestyle interventions. The following principles shift the emphasis towards motivational abilities and can be applied as preventative measures at any stage of life.

- Challenges should not be avoided. We should find solutions to problems that at first seem insurmountable.

- Self-commitment ('Do not give up') and conscientiousness intensify the experiences we have, and although these are sometimes difficult to sustain, the outcome can be very rewarding and instructive for the future.

- The capacity to regulate ourselves and overcome impulses and drives that tempt us - to overeat, drink too much alcohol, take harmful drugs, say hurtful things to others - should be enhanced. This has a short- and a long-term perspective. Self-regulation may fail now and again; the important thing is to regain the former degree or a better level of control and not to give up (so-called ego-depletion ${ }^{15}$ ).

- We should make ourselves repeatedly aware of our purpose in life by active reflection. The self-coherence thus achieved seems to benefit psychological processes and the brain. ${ }^{16}$

Although these principles at present look more like the idealistic hopes of a self-advancement movement, we would like to express our cautious optimism that they may one day be tested empirically for the benefit of patients, in the same way as cognitive variables have been.

\section{About the authors}

Andreas Maercker is Professor of Psychopathology and Clinical Intervention, Simon Forstmeier is Senior Teaching and Research Associate, University of Zurich, Zurich, Switzerland.

\section{References}

1 Valenzuela MJ. Brain reserve and the prevention of dementia. Curr Opin Psychiatry 2008; 21: 296-302.

2 Forstmeier S, Maercker A. Motivational reserve: lifetime motivational abilities contribute to cognitive and emotional health in old age. Psychol Aging 2008; 23: 886-99.
3 Forstmeier S, Maercker A. Potentially modifiable risk factors in the development of Alzheimer's Disease. Eur Neurol Rev 2009; 4: 18-21.

4 Mischel W, Ebbesen EB, Zeiss AR. Cognitive and attentional mechanisms in delay of gratification. J Pers Soc Psychol 1972; 21: 204-18.

5 Mischel W, Ayduk O, Berman MG, Casey BJ, Gotlib IH, Jonides J, et al. 'Willpower' over the life span: decomposing self-regulation. Soc Cogn Affect Neurosci 2011; 6: 252-6.

6 Forstmeier S, Drobetz R, Maercker A. The delay of gratification test for adults: validating a behavioral measure of self-motivation in a sample of older people. Motiv Emot 2011; 35: 118-34.

7 Peterson NG, Mumford MD, Borman WC, Jeanneret PR, Fleishman EA An Occupational Information System for the 21st Century: The Development of $0^{\star} N E T$. American Psychological Association, 1999.

8 Forstmeier S, Maercker A, Maier W, van den Bussche H, Riedel-Heller S, Kaduszkiewicz $\mathrm{H}$, et al. Motivational reserve: motivation-related occupational abilities and risk of mild cognitive impairment and Alzheimer disease. Psychol Aging 2011; 29 Aug [Epub ahead of print].

9 Luck T, Riedel-Heller SG, Kaduszkiewicz H, Bickel H, Jessen F, Pentzek $M$, et al. Mild cognitive impairment in general practice: agespecific prevalence and correlate results from the German study on ageing, cognition and dementia in primary care patients (AgeCoDe). Dement Geriatr Cogn Disord 2007; 24: 307-16.

10 Polidori MC, Nelles G, Pientka L. Prevention of dementia: focus on lifestyle. Int J Alzheimers Dis 2010; pii: 393579.

11 Wilson RS, Schneider JA, Arnold SE, Bienias JL, Bennett DA Conscientiousness and the incidence of Alzheimer disease and mild cognitive impairment. Arch Gen Psychiatry 2007; 64: 1204-12.

12 Pocnet C, Rossier J, Antonietti JP, von Gunten A. Personality changes in patients with beginning Alzheimer disease. Can J Psychiatry 2011; 56: 408-17.

13 Boyle PA, Buchman AS, Barnes LL, Bennett DA. Effect of a purpose in life on risk of incident Alzheimer Disease and mild cognitive impairment in community-dwelling older adults. Arch Gen Psychiatry 2010; 67: 304-10.

14 Wollen KA. Alzheimer's disease: the pros and cons of pharmaceutical, nutritional, botanical, and stimulatory therapies, with a discussion of treatment strategies from the perspective of patients and practitioners. Altern Med Rev 2010; 15: 223-44.

15 Hagger MS, Wood C, Stiff C, Chatzisarantis NL. Ego depletion and the strength model of self-control: a meta-analysis. Psychol Bull 2010; 136 495-525.

16 Freund AM, Nikitin J, Ritter JO. Psychological consequences of longevity: the increasing importance of self-regulation in old age. Human Dev 2009; 52: 1-37. 